\title{
A walk on the wild side
}

\section{Dustin M. Graham}

Dirty mice are helping researchers clean up translational science, but their wild ways create risks for institutions more accustomed to the prim and proper.

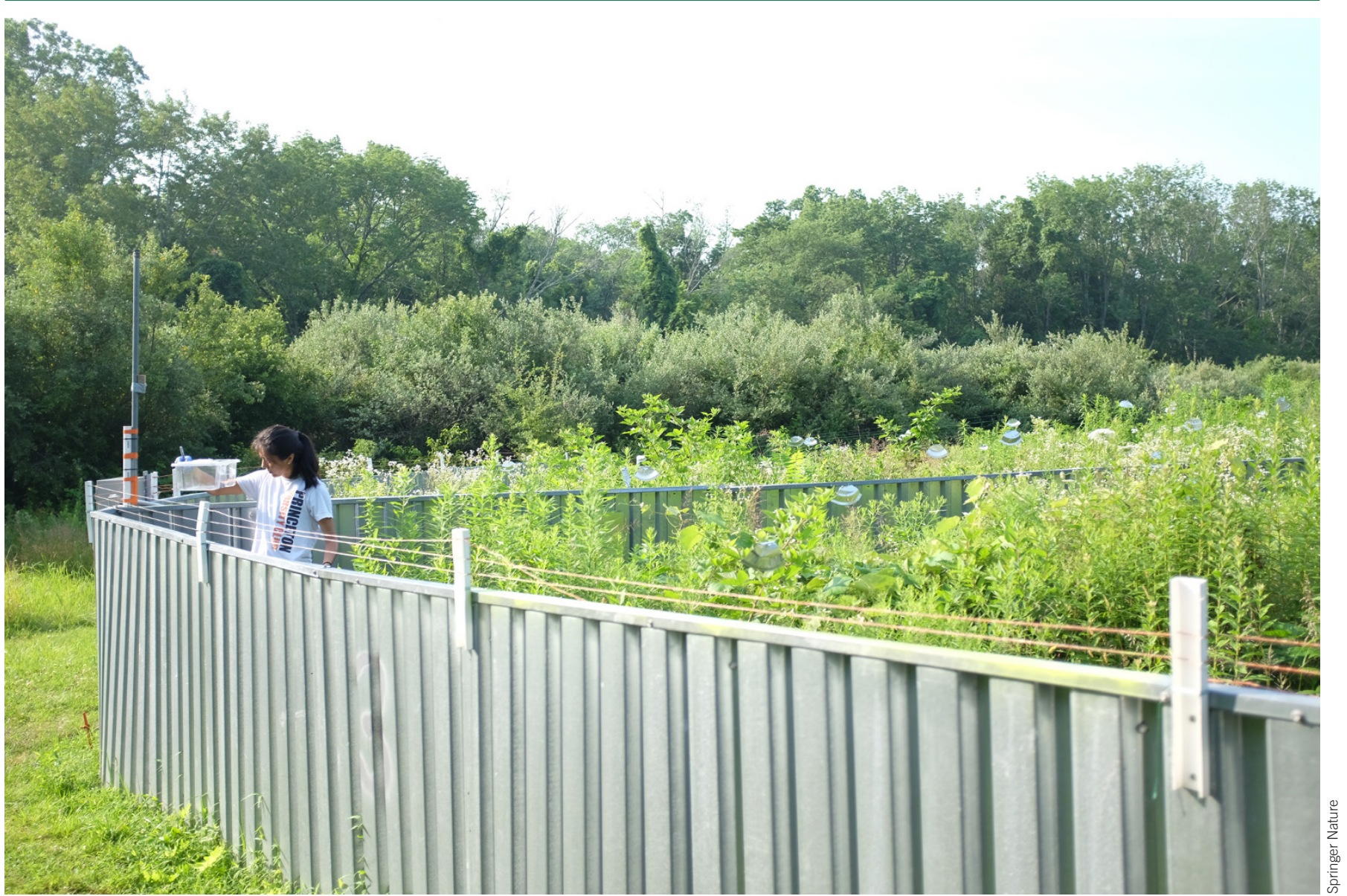

For most immunologists, the big red barn that sits on a farm near Princeton, New Jersey, would be an odd place to store data. It has no lab benches or freezers. The inside walls are covered in cobwebs, and overhead, dusty blue tarps stretch across the rafters, arched downwards from the weight of petrified bat guano. But standing on the barn's floor is a server, humming away as it collects wireless data from a unique source outside. The source is a circular 1,500 square-meter open-air enclosure sitting in a field at the farm's edge. This is where the wild(ish) things are, and where Andrea Graham is about to start the safari.

Graham, an evolutionary ecologist at Princeton University, hops over the enclosure's fence and gathers her students for their weekly reconnaissance. Walking through the weeds, she inspects the Longworth traps her team laid out the night before, seeing if any have been tripped. Longworth traps are designed for behavioral ecologists to capture, without injury, small wild mammals, such as mice. But as Graham explains, the mice they are searching for on this farm aren't exactly wild, not yet at least. "These guys were bred in a lab at New York University, actually, so they're big city mice." Spotting a trap with some movement inside, Graham scans the first catch of the day, making use of the radio-frequency chip implanted in each mouse for tracking.

“Mouse 51-51-57," Graham announces.

"We caught it last time," says Sriveena Chittamuri, a visiting undergraduate. "We have enough poop samples, but we still need urine." 


\section{TECHNOLOGY FEATURE}

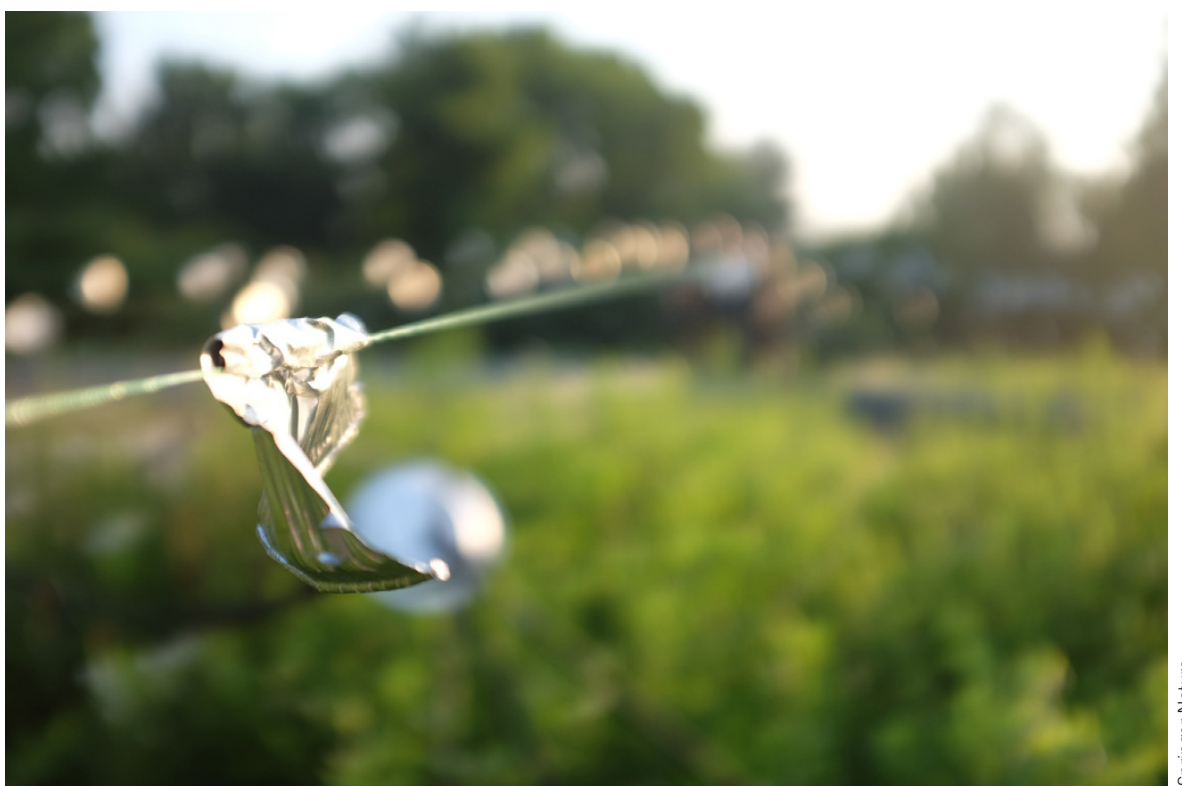

SAFE HAVEN | Pie plates tied across strings above the outdoor enclosure help keep away birds.

The group carefully moves the mouse to a clean cage to collect fresh excreta for microbiome analysis. Afterwards, they open the cage, and the mouse, no worse for the wear, scampers back into the weeds that are full of life, but free of predators. Graham says that for the next two months, she and her students will repeat this unique 'catch-and-release' strategy in the outdoor enclosure with the same group of lab mice. Her students will collect samples from the mice every 10-14 days, to quantify changes in the mice's microbiomes after exposure to a microbial world they have never seen before, but one that most other animals, including humans, come into contact with everyday.

"We're basically asking, how rapidly can we convert a hygienic lab mouse into a more natural mouse," says Graham. "We call it 'rewilding."

By taking lab mice from Manhattan and setting them free on a farm in New Jersey, Graham hopes to better understand how changes in the microbiome can impact immune system mechanisms regulating parasitic worm infections. More broadly, she wants to see if adding a touch of the real world into her immunology experiments can affect genotype-phenotype relationships in mouse models of human disease, which could reveal their potential for translatability. But given the controlled confines that biomedical research typically operates with-
Nature, their teams compared the immune systems of clean lab mice with dirty pet shop mice and found that not only did pet shop mice have immune systems that better mimicked those of adult humans, but when used as 'dirty roommates', pet shop mice could transfer their adult human-like immune system phenotype to previously clean lab mice, demonstrating a role for the microbiome in driving the changes ${ }^{1}$.

Eleanor Riley, Director of the Roslin Institute at the University of Edinburgh, calls results like these "immunology's dirty little secret." For years she's done research on infectious disease in humans and also in lab mice, and has been "aware for a while that the results you get in mice, just like in humans, really depends on where they come from." In a recent paper with collaborator and helminth parasitemia expert Mark Viney, from the University of Bristol, she looked at the immune systems of feral mice from all over the UK-including farms and the London Underground-and compared them with clean lab mice ${ }^{2}$. In line with Masopust's and Jameson's work, they found several quantitative and qualitative differences in dirty (wild) vs. clean (lab) mice immune systems, differences that she believes need to be taken into account by more translational scientists. "We see in humans that a difference in environments really helps to calibrate our

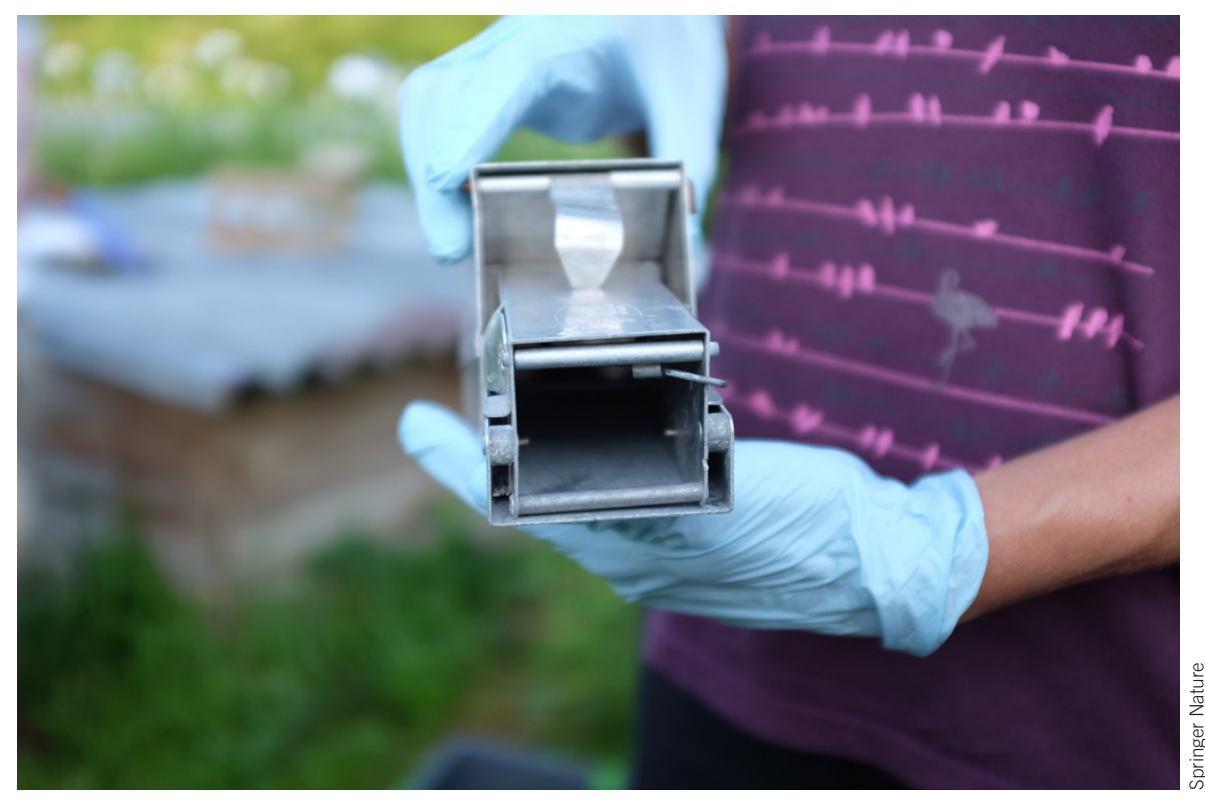

TRAPPER KEEPER | An example Longworth trap used for catching mice in the enclosure. 


\section{TECHNOLOGY FEATURE}

immune response, and that's exactly what we're seeing in lab vs. wild mice. And so by extension, when you're translating a drug from a lab mouse to a human, you're jumping from very clean to dirty environments, as well as across species, and I think that complicates the translation." Adding some studies with dirty animals before clinical trials, Riley says, could help spot treatments earlier in the pipeline that work only under artificially neat and tidy conditions; conditions unlikely to be encountered by most humans.

But even if adding a little dirt can go a long way towards improving the translatability of disease model research, it's difficult to know how it will impact a study's reproducibility, another hot-button issue in biomedical research. "Everyone wants reproducible results," Riley says, which usually means eliminating variables, not adding more of them. Biomedical research has adopted the reductionist approach with great success, she says, and going out into the wild (or bringing it into the lab) might help to improve translation, but needs to be balanced with reproducible outcomes.

"There's a kind of tension between getting highly reproducible data, which I absolutely understand, and the need to have a more real-world solution."

Nowhere is that tension greater than in the animal care facilities that house the models used for research. After thirty years and millions of dollars spent cleaning up these facilities, institutions are hesitant to roll out the red carpet for dirty animals.

\section{A culture of clean}

When Masopust's and Jameson's pet shop paper came out in Nature, says Ken Boschert, Associate Director of Comparative Medicine at Washington University, he and other university veterinarians began receiving requests from investigators to start bringing in similarly dirty mice into animal care facilities. "We're all kind of shaking our heads right now and trying to figure out what's the best way to deal with this," says Abigail Smith, Professor of Pathobiology at University of Pennsylvania School of Veterinary Medicine, "because indeed, I heard almost immediately from investigators at the medical school that contacted me about this."

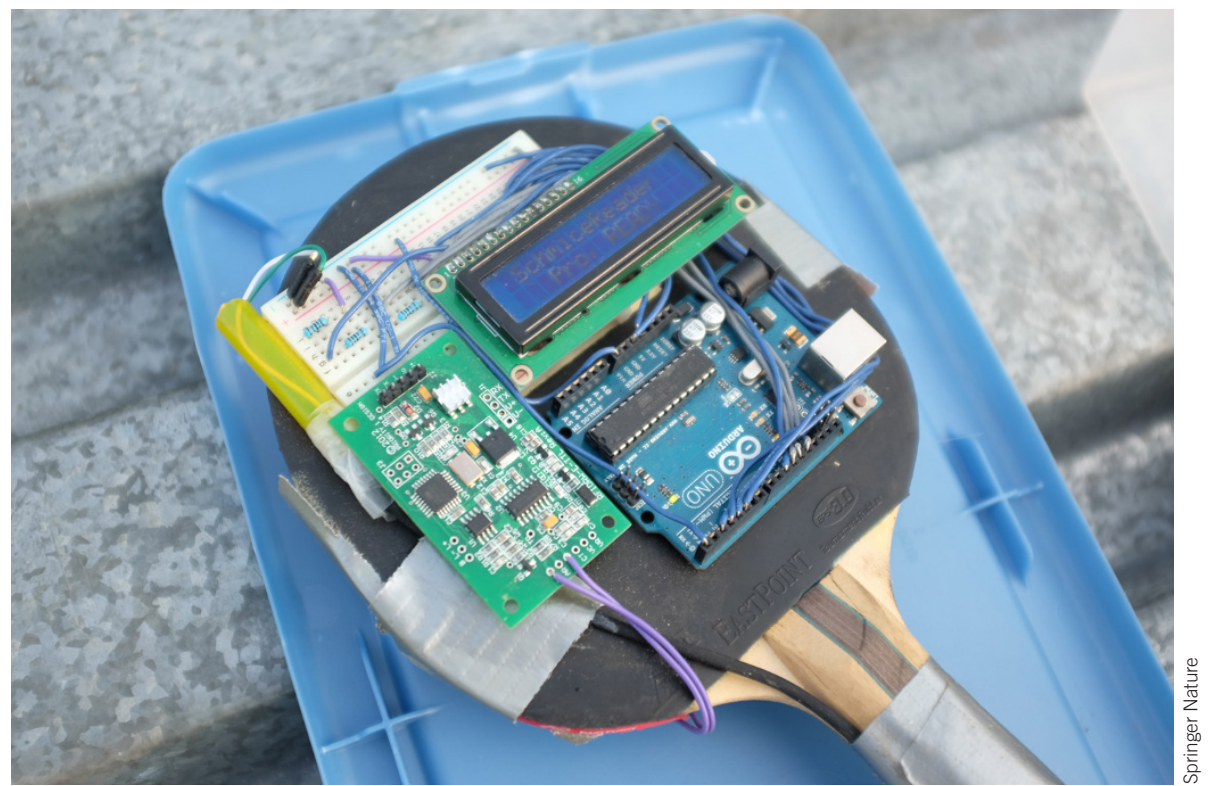

TOOLS OF THE TRADE | A homemade RFID scanner (invented by Quentin Caudron and Romain Garnier) used by Graham's group to identify tagged mice on the farm.

Boschert, Smith, and other university veterinarians have done their best to accommodate a handful of studies with pet shop or otherwise contaminated miceJameson and Masopust, for instance, coordinated with veterinary staff at University of Minnesota to use a BSL-3 room for their study-but stop-gap measures can only go so far, and facility directors have to consider the hundreds of thousands of clean mice under their care that could be susceptible to infections. "There's no way I'm going to expose all of them, it would be insanity," says Boschert. "You can't destroy the value of your mice."

Boschert has good reason to be concerned about the growing interest in dirty mice. He can recall a time in decades past-a time Boschert refers to as the "wild west"- when veterinarians were trying to care for rodent models with highly variable health statuses. "Thirty years ago there was little quality control at universities or at vendors. You'd receive your animals, maybe see a few deaths early on, and then boom, a wave of infections and animals gone to waste." In addition to poor welfare, Boschert says this laissez faire approach to microbes also made for bad science. But the key change to improving the cleanliness and consistency of animals had to start from rodent vendors. "No matter how clean we made our facilities," Boschert says, "if all the animals came in dirty, there was nothing we could do about it. It was really the vendors cleaning up their act that got things started."

These days, the approach to rodent health status is very different, says Smith. All of the major mouse vendors provide specific pathogen free (SPF) animals, and most research universities have hygienic barrier facilities to keep contamination of colonies down to a minimum (see also Box 1). They've also developed strict standard operating procedures for biocontainment. At University of Pennsylvania, some animal care areas require staff to wear colorcoded personal protective equipment, so everyone can easily see who's allowed to go where, limiting the risk of people accidentally dragging bugs into rooms with different containment levels.

Many of the changes made by institutions over the years tend to come from learning things the hard way. One research university veterinarian described an outbreak three years ago in their facility that cost hundreds of thousands of dollars in ruined mice-not to mention the months of downtime to get the room decontaminated-a story all too common in many facilities. Embracing dirty mice on a larger scale, therefore, could come with significant risks that veterinarians like Boschert and Smith still need to evaluate. 


Box 1 Some example
recommended infectious
agents to monitor in
mice (see ref. 6)
Viruses
Mouse hepatitis virus
Mouse hepatitis virus
Mouse rotavirus
Murine norovirus
Parvoviruses:
Minute virus of mice
Mouse parvovirus
Theiler's murine encephalomyelitis virus
Lymphocytic choriomeningitis virus
Mouse adenovirus type 1 (FL)
Mouse adenovirus type 2 (K87)
Mousepox (ectromelia) virus
Pneumonia virus of mice
Reovirus type 3
Sendai virus
Bacteria
Helicobacter spp.
Pasteurella pneumotropica
Streptococci b-haemolytic (not group D)
Streptococcus pneumoniae
Citrobacter rodentium
Clostridium piliforme
Corynebacterium kutscheri
Mycoplasma pulmonis
Salmonella spp.
Streptobacillus moniliformis

How then do veterinarians and investigators balance the need for clean living in mouse facilities with the dirty truth of translational science?

\section{Redefining dirty}

One solution, Graham says, is to view the concepts of dirty or wild in the lab as a spectrum. "I'm well aware that putting animals on a farm or bringing in pet shop mice are pretty dramatic approaches." She envisions some researchers taking smaller steps to 'wildize' their mice, like adding some outdoor soil into their cages, that wouldn't be too difficult or risky for facilities to implement.

Another approach, favored by Andrew Macpherson, Professor of Medicine and Director of Gastroenterology at the University Hospital of Bern, is to develop lines of mice in a similar vein to inbred isogenic mouse strains, but applied to microbiota. Akin to the many isogenic strains investigators pick and choose from, like C57BL/6 or BALB/c, these 'isobiotic' strains would be diverse-C57BL/6 with microbiota X, C57BL/6 with microbiota Y, BALB/c with microbiota Z...- and provide investigators with animals having a stable microbiome, generation to generation, and most importantly, institution to institution. "The vision is that these sorts of models should be as diverse and as freely available as the isogenic models are today," Macpherson says. His group has recently developed an isobiotic mouse line named "stable defined moderately diverse microbiota in the specific-pathogen-free mouse 2" (sDMD$\mathrm{Mm} 2$ ) which recapitulates immune system adaptations and disease model phenotypes of the conventional mouse lines that have much more complex and undefined microbiomes ${ }^{3}$. The sDMDMm2 microbiota are stable across generations, but only when the mice are maintained in ultra-clean gnotobiotic conditions. Macpherson acknowledges that a major challenge will be maintaining the microbiome stability of sDMDMm2 mice under more typical and far less costly housing conditions, like individual ventilated cages.

Yet another solution, says Peter Smith, Associate Professor of Comparative Medicine at Yale, is for investigators to make use of the varying degrees of 'dirty' that are already present at different institutions, by regularly sharing knowledge about the differences in what microbes their institutions do and do not allow into facilities. This could be done by including health status reports in publications, but investigators rarely provide this kind of information, creating trouble for researchers who want to compare their disease model phenotype with the phenotype of a similar model used at another institution. Without knowing what microbes were allowed or not allowed in those colonies, it's nearly impossible to understand the reasons for discrepancies in results.

Although Yale's Smith agrees that all of the effort to clean up facilities has helped improve mouse research, he does wonder whether it's possible to go too far in trying to create a strict culture of clean. "I think we need to start redefining 'dirty' as something that isn't necessarily bad under all conditions, so that investigators are more open to reporting it."

\section{Thunderstruck}

Ken Cadwell, Associate Professor in the Department of Microbiology at the New York University School of Medicine (NYUSOM), knows first hand how differences in an institution's panel of microbes can impact disease model research. But rather than bemoan it, he's taken advantage of it. As a postdoctoral researcher working on mouse models of inflammatory bowel disease (IBD) in Herbert Virgin's lab at Washington University, he discovered that murine norovirus - a common mouse virus that some university facilities allow and some exclude-was a trigger for the IBD phenotype in a Atg16L1 mutant mouse line that models Crohn's disease ${ }^{4}$. "It was only when we cleaned the mice up that we realized murine norovirus was causing intestinal pathologies," says Cadwell.

Working on another mouse model of IBD-Nod 2 knockout mice-but this time at his own lab at NYUSOM, his group found that the IBD phenotype in -/-Nod2 mice was dependent on the bacterial species Bacteroides vulgatus, which again, some institutions like NYUSOM have in their facilities, and some do not ${ }^{5}$. "So lightning struck twice," says Cadwell. "This is the second example of something being a little bit different in one animal facility that is essential for the disease manifestations to occur in the mouse model." While results like these are exciting, Cadwell says that relying on happenstance for these types of discoveries might not be the best method.

Together with colleague P'ng Loke, Associate Professor and helminth parasitologist at NYUSOM, they're teaming up with Graham at the farm in New Jersey to see how far they can push the -/-Nod2 IBD phenotype out in the wild, and test how natural microbes influence their mutant mice. Graham says it's essentially looking at the 'hygiene hypothesis' - which links a lack of early childhood exposure to natural microbes with increased risk for disorders like IBD - in mice, but in reverse, since the mice are born into hygienic facilities and are then moved out into the dirty field as adults. Although it will take some time, Graham says, the research team hopes that in addition to testing how well the IBD phenotypes in their mouse models hold up on the farm, they can also combine different methods to try and establish mechanistic causes for any changes they see. 


\section{Old friends}

Back at the farm and sitting in the cleanup area by the enclosure, Graham and her team scrub down the traps and the cages, getting ready for another day's safari with mice that will be a bit closer to the wild things their kind used to be. As Graham talks about the translatability of lab-based phenotypes and the intricacies of IACUC protocols for rodents living in the outdoors, she also mentions the farm's ability to turn back the clock on her team's immunology studies with mice, to a more wild time.
"Once they're out on this farm, it's basically the evolutionary natural setting for this commensal Mus musculus," says Graham.

She admits that challenges remain for wildizing lab mice and applying a multidisciplinary 'eco-immunology' approach to translational research. But as the server in the barn continues to receive data from tagged mice in the enclosure-their feeding habits, their comings-and-goings-Graham is eager to see not just how well their phenotypes might translate to humans, but how these mice will cope with microbes and conditions that their pre-lab ancestors lived with, and indeed, evolved their immune systems with.

Lab mice, welcome to the farm. Meet your new old friends.

1. Beura, L.K. et al. Nature 532, 512-516 (2016).

2. Abolins, S. et al. Nat. Commun. 8, 14811 (2017).

3. Uchimura, Y. et al. 4, e00951-16 (2016)

4. Cadwell, K. et al. Cell 141, 1135-1145 (2010).

5. Ramanan, D. et al. Immunity, 41, 311-324 (2014).

6. FELASA working group on revision of guidelines for health monitoring of rodents and rabbits $L a b$ Anim. 48, 178-192 (2014). 